\title{
Combination measles-mumps-rubella-varicella vaccination and the risk of febrile seizure
}

\author{
Karina A. Top MD MSc, Noni E. MacDonald MD MSc
}

See related research article on page 824 and at www.cmaj.ca/lookup/doi/10.1503/cmaj.140078

Competing interests: None declared.

This article was solicited and has not been peer reviewed.

Correspondence to: Karina Top, karina.top@ dal.ca

CMAJ 2014. DOI:10.1503/ cmaj.140778
$\mathrm{V}$ accination programs are a highly effective public health intervention, having led to dramatic reductions in childhood morbidity and mortality. However, as the recent measles outbreaks in Canada have illustrated, ${ }^{1}$ control of vaccine-preventable diseases can be maintained only through continued high public acceptance of - and confidence in - vaccination programs. Postlicensure surveillance and evaluation of vaccine safety are two key components for maintaining public confidence in vaccines and ensuring that vaccination programs continue to provide the maximal benefit to society at minimal risk.

In a related article in CMAJ, MacDonald and colleagues $^{2}$ report the findings of their population-based cohort study involving nearly 280000 children in Alberta in which they compared the risk of seizures in children 12-23 months of age who received the combination measles-mumps-rubella-varicella (MMRV) vaccine (Priorix-Tetra, GlaxoSmithKline) with the risk in those who received separate MMR and varicella $(M M R+V)$ vaccines at the same visit. The study period from 2006 to 2012 spans the switch from separate MMR+V vaccines to the combination MMRV vaccine in 2010. The MMR vaccine is known to be associated with the phenomenon of febrile seizures, which are brief $(\leq 15 \mathrm{~min})$, generalized convulsions that occur in association with fever in children six months to five years of age. ${ }^{3,4}$ Although

\section{- Ker Points}

- A population-based cohort study from Alberta involving children 12-23 months of age reported an excess risk of febrile seizure after measles-mumps-rubella-varicella (MMRV) vaccination of 1 per 2841 doses, compared with separate MMR and varicella (MMR+V) vaccinations.

- These findings support those from other countries using different formulations of MMRV.

- More research is needed to determine the optimal schedule for MMR and varicella vaccination that balances risks, benefits, costs, parental attitudes and public acceptability, and Canada is in a unique position to address these issues.

febrile seizures are generally benign and are not associated with long-term neurologic sequelae or developmental delay, they often frighten parents and prompt visits to the emergency department. ${ }^{3}$ The peak risk of fever after MMR coincides with the peak in viral replication on days 7-10 after vaccination; fever occurs in about $10 \%$ of children receiving the vaccine, of whom $0.3 \%-0.4 \%$ will have a febrile seizure. ${ }^{4,5}$ Fever and febrile seizure occur more commonly following the first dose than the second. ${ }^{5}$

MacDonald and colleagues ${ }^{2}$ compared the risk of ambulatory and inpatient visits for seizure 7-10 days after vaccination with the risk in the 42 days before vaccination. The authors determined that the relative risk of seizure was increased on days 7-10 in both the MMRV and $\mathrm{MMR}+\mathrm{V}$ groups, as would be expected. However, the risk was twice as high after MMRV than after MMR+V (relative risk 1.99, 95\% confidence interval $[\mathrm{CI}] 1.30-3.05$, adjusted for age and calendar year). This translates to one additional febrile seizure for every $2841 \mathrm{MMRV}$ doses administered. Of note, in children with a history of seizure or a seizure disorder, there was no difference in risk between the MMRV and $\mathrm{MMR}+\mathrm{V}$ vaccines (relative risk $1.30,95 \% \mathrm{CI}$ $0.60-2.79)^{2}$

These findings are similar to those of population-based cohort studies conducted in the United States with a different MMRV formulation, ProQuad (Merck), which showed an excess risk of one febrile seizure per about 2300-2600 doses of MMRV, compared with separate MMR+V vaccines. ${ }^{4,6}$ MacDonald and colleagues' ${ }^{2}$ study is the first in Canada, and the largest study to date to assess this risk with the Priorix-Tetra formulation of MMRV. As the authors discuss, a recent matched-cohort study from Germany reported a 3.5-fold increase in febrile seizure on days 5-12 after vaccination with Priorix-Tetra (MMRV) versus $\mathrm{MMR}+\mathrm{V}$ (odds ratio 3.5, 95\% CI $0.7-$ 19.0). ${ }^{7}$ The lack of a significant difference may be due to the inclusion of only patients admitted to hospital and of only children over four years of 
age, in whom the risk of febrile seizure after MMRV is not increased. ${ }^{8}$

In discussions of the risks of febrile seizure associated with MMR and MMRV, it is important to note that these risks are far lower than the risk of complications from measles, which occur in about $10 \%$ of cases. ${ }^{5}$ However, febrile seizures, although benign, are concerning to many parents. Some may perceive the risks of the vaccine as higher than the risks of disease. The health care costs associated with febrile seizures must also be considered when deciding which vaccine to use in children 12-23 months of age. As MacDonald and colleagues point out, the small increase in the risk of febrile seizures with MMRV must be balanced against the benefits of a single, combined vaccination that reduces the number of injections young children will require, the latter a concern for many parents. Canadian data are lacking regarding parental attitudes and perceptions of the risks and benefits of MMRV versus $\mathrm{MMR}+\mathrm{V}$. This is therefore an important research priority to ensure policies are based on the best evidence.

In countries currently vaccinating children against varicella, further research is needed to determine the optimal schedule for $\mathrm{MMR}+\mathrm{V}$ vaccination. Canada is uniquely positioned to evaluate the safety and effectiveness of different MMR, varicella and MMRV schedules because of the 13 different vaccination schedules used across the country, one for each province and territory (Appendix 1, available at www.cmaj.ca/lookup/ suppl/doi:10.1503/cmaj.140778/-/DC1). ${ }^{9}$ Seven provinces currently administer MMRV at 12 months of age, and four administer MMRV as the second dose at 18 months of age. Data are lacking on the safety and acceptability of several of these schedules.

A hybrid program of separate $\mathrm{MMR}+\mathrm{V}$ vaccines for the first doses, followed by MMRV for the second dose may end up achieving the optimal balance of risks and benefits. This approach may be more costly to the public health system because of the need to purchase MMR, varicella and MMRV vaccines, but the costs may be offset by decreased emergency visits for febrile seizure and improved confidence in vaccination. Decision-making regarding $\mathrm{MMR}+\mathrm{V}$ vaccination will thus benefit from further research in three key areas: evaluation of vaccine safety, public acceptability of the risks and benefits of the different schedules, and cost-effectiveness of the different schedules.

\section{References}

1. Deehan H, Shane A. Measles activity in Canada: January - June 2014. Can Commun Dis Rep 2014;40-12:233-5.

2. MacDonald SE, Dover DC, Simmonds KA, et al. Risk of febrile seizures after first dose of measles-mumps-rubella-varicella vaccine: a population-based cohort study. CMAJ 2014 June 9 [Epub ahead of print].

3. Friedman MJ, Sharieff GQ. Seizures in children. Pediatr Clin North Am 2006;53:257-77.

4. Klein NP, Fireman B, Yih WK, et al. Measles-mumps-rubellavaricella combination vaccine and the risk of febrile seizures. Pediatrics 2010;126:e1-8.

5. Strebel PM, Papania MJ, Parker Fiebelkorn A, et al. Measles vaccine. In: Plotkin SA, Orenstein WA, Offit PA, editors. Vaccines. 6th ed. Philadelphia (PA): Saunders Elsevier; 2013.

6. Jacobsen SJ, Ackerson BK, Sy LS, et al. Observational safety study of febrile convulsion following first dose MMRV vaccination in a managed care setting. Vaccine 2009;27:4656-61.

7. Schink T, Holstiege J, Kowalzik F, et al. Risk of febrile convulsions after MMRV vaccination in comparison to MMR or MMR+V vaccination. Vaccine 2014;32:645-50.

8. Klein NP, Lewis E, Baxter R, et al. Measles-containing vaccines and febrile seizures in children age 4 to 6 years. Pediatrics 2012;129:809-14.

9. Publicly funded immunization programs in Canada - routine schedule for infants and children including special programs and catch-up programs (as of March 2014). Ottawa: Public Health Agency of Canada; 2014. Available: www.phac-aspc.gc.ca/im/ ptimprog-progimpt/table-1-eng.php (updated 2014 Mar. 17; accessed 2014 June 12).

Affiliations: Department of Pediatrics (Top, MacDonald) and Department of Community Health \& Epidemiology (Top), Dalhousie University, Halifax, NS

Contributors: Karina Top drafted the article, which both authors revised and approved for publication.

\section{Call for papers: CMAJ Holiday Reading}

'Twas months before the holidays and all through CMAJ house not a submission was stirring, making editors grouse. "Holiday Reading time is nigh!" they cried in despair, in hopes that your papers soon would be there.

Submit your brilliant missives, rigorous research (based on real data) on quirky topics or holiday-themed visuals at http://mc.manuscriptcentral.com/cmaj. Nonresearch submissions should be no longer than 1200 words. For information, contact kelly.clarke@cmaj.ca.

Deadline: October 1, 2014

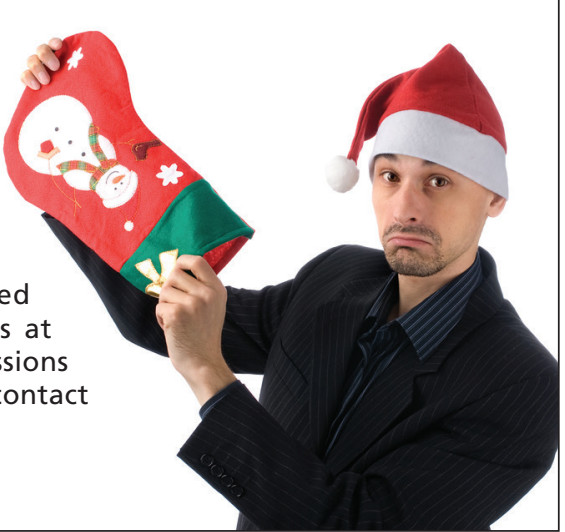

